\title{
Locally solvable subnormal and quasinormal subgroups in division rings
}

\author{
Le Qui Danh and Huynh VIET KHANH \\ (Received April 23, 2020) \\ (Revised March 4, 2021)
}

\begin{abstract}
In this paper, we show that every locally solvable subnormal subgroup or locally solvable quasinormal subgroup of the multiplicative group of a division ring is central.
\end{abstract}

\section{Introduction}

A subgroup $N$ of a group $G$ is said to be subnormal in $G$ if there is a finite chain of subgroups

$$
N=N_{r} \leq N_{r-1} \leq \cdots \leq N_{0}=G
$$

for which $N_{i}$ is normal in $N_{i-1}$ for all $1 \leq i \leq r$. Also, if $Q$ is a subgroup of $G$ such that the relation $Q H=H Q$ holds for any subgroup $H$ of $G$, then we say that $Q$ is quasinormal (or permutable) in $G$. It is pointed out in [11, Chapter 7] that there are close relations between these types of subgroups. It was shown by S. E. Stonehewer that if $G$ is a finitely generated group, then every quasinormal subgroup of $G$ is subnormal ([12, Theorem B]). However, the converse does not hold up. As an example, let $G$ be the dihedral group of order 8 generated by subgroups $A$ and $B$ which are of order 2 . It follows that $A B \neq B A$ since $|A B|=4$ and $G \neq A B$, implying that $A$ and $B$ are not quasinormal subgroups of $G$. On the other hand, the nilpotency of $G$ implies that both $A$ and $B$ are subnormal. (Recall that every subgroup of a nilpotent group is subnormal.) In this paper, we study subnormal subgroups and quasinormal subgroups of the multiplicative group of a division ring. Relating to this, note that in [1] there is an example of a division ring which contains quasinormal subgroups that are not subnormal.

The second author is funded by Vietnam National University HoChiMinh City (VNUHCM) under grant number B2020-18-02.

2010 Mathematics Subject Classification. Primary 16K20, 20F19.

Key words and phrases. division ring, locally solvable subgroup, quasinormal subgroup, subnormal subgroup. 
In the literature, there are very rich results concerning the algebraic structure of multiplicative subgroups in a division ring (e.g., see [6]). As a direction of the study, in 1950's and 1960's, many authors paid attention to an interesting question of how far the multiplicative group $D^{*}$ of a division ring $D$ is from being abelian. In this direction, a well-known result of L. K. Hua says that if $D^{*}$ is solvable, then $D$ is a field. Motivated by this result, several other authors examined various aspects of subnormal subgroups of $D^{*}$, instead of the whole group $D^{*}$. For example, it was shown that every subnormal subgroup of $D^{*}$ must be central in $D$ if it is locally nilpotent, solvable, or $n$-Engel (see [13], [8], [10], respectively). Now, we consider the same problem in which the subnormal subgroup is assumed to be locally solvable. By definition, a group is called locally solvable if its every finitely generated subgroup is solvable. In [4], B. X. Hai and D. V. P. Ha proved that if $D^{*}$ is locally solvable, then $D$ is a field. Moreover, it was proved by A. E. Zalesskii in [17] that every locally solvable normal subgroup of $D^{*}$ is contained in the center $F$ of $D$. It is natural to ask whether every locally solvable subnormal subgroup, say $G$, of $D^{*}$ is also contained in $F$. A positive answer to this question was given for some particular cases where $D$ is supposed to be algebraic over $F$ ([5]), or where the derived subgroup $G^{(i)}$ of $G$ is assumed to be algebraic over $F$ for some $i \geq 1([9])$. The first purpose of the present paper is to give the affirmative answer to the question in the general setting; that is, we shall show that every locally solvable subnormal subgroup of $D^{*}$ is contained in $F$ (Theorem 1). The second purpose is to prove that every locally solvable quasinormal subgroup of $D^{*}$ is also central; and this goal will be achieved in Theorem 3.

Throughout this paper, the word "ring" always refers to a ring with an identity element $1 \neq 0$. For a ring $R$, the symbol $R^{*}$ denotes the group of units in $R$. If $D$ is a division ring with center $F$ and $S$ is a subset of $D$, then $F[S]$ (resp. $F(S)$ ) denotes the subring (resp. the division subring) of $D$ generated by $F \cup S$. For a group $G$, the Hirsch-Plotkin radical of $G$ is defined to be the subgroup generated by all locally nilpotent normal subgroups of $G$. If $H$ and $K$ are two subgroups of $G$, then the symbol $[H, K]$ stands for the subgroup of $G$ generated by the set of all commutators $[a, b]=a^{-1} b^{-1} a b$, where $a \in H$ and $b \in K$. We say that $G$ is radical over a subgroup $Q$ if for each $g$ in $G$, there is a positive integer $n$ depending on $g$ such that $g^{n}$ belongs to $Q$.

\section{Locally solvable subnormal subgroups}

We begin with a group-theoretic lemma which, despite its apparent simplicity, will be frequently applied in the sequel. 
Lemma 1. Every group contains a unique maximal periodic normal subgroup. Moreover, such a subgroup is characteristic in the whole group.

Proof. Our proof shall be obtained by mainly using Zorn's Lemma. First, we define a family of subgroups of a group $G$ by taking

$$
\mathscr{A}=\{H \mid H \text { is a periodic normal subgroup of } G\} .
$$

This family is obviously non-empty since the identity subgroup belongs to $\mathscr{A}$. Now, we consider an arbitrary chain $\left\{H_{i}\right\}$ of subgroups in $\mathscr{A}$. Our task, of course, is to show that $\bigcup H_{i}$ is again a member of $\mathscr{A}$; that is, to prove that $\bigcup H_{i}$ forms a periodic normal subgroup of $G$. For this purpose, pick any two elements $a, b \in \bigcup H_{i}$. Then, there exist indices $i$ and $j$ for which $a \in H_{i}$ and $b \in H_{j}$. Since the collection $\left\{H_{i}\right\}$ forms a chain, either $H_{i} \subseteq H_{j}$ or $H_{j} \subseteq H_{i}$. It is clear that we may assume that $H_{i} \subseteq H_{j}$ and so $a \cdot b^{-1} \in H_{j} \subseteq \bigcup H_{i}$. This implies that $\bigcup H_{i}$ is a subgroup of $G$. The normality as well as the periodicity of $\bigcup H_{i}$ may be obtained by the same way. All of this shows that $\bigcup H_{i}$ is a member of $\mathscr{A}$, completing our task. Therefore, on the basic of Zorn's Lemma, the family $\mathscr{A}$ contains a maximal element $M$.

Next, we shall prove that $M$ is maximal with respect to being periodic and normal. Let $N$ be a periodic normal subgroup of $G$ for which $M \subseteq N$. Since $M$ is a maximal element of $\mathscr{A}$ and $N \in \mathscr{A}$, we must have $M=N$, which implies the maximality of $M$.

To see the uniqueness of $M$, take any periodic normal subgroup $A$ of $G$. The normality of $M$ and $A$ in $G$ permits us to form the product subgroup $A M$, which is obviously a periodic normal subgroup of $G$. But then, the maximality of $M$ reveals that $A M=M$, or $A \subseteq M$. This argument shows that every periodic normal subgroup of $G$ is contained in $M$, proving the uniqueness of $M$.

It remains only to show that $M$ is characteristic in $G$. For this purpose, we pick $\varphi \in \operatorname{Aut}(G)$, then $\varphi(M)$ is certainly a periodic normal subgroup of $G$. The uniqueness of $M$ implies that $\varphi(M)=M$. Our proof is finally finished.

For any group $G$, let us denote by $\tau(G)$ the unique maximal periodic normal subgroup of $G$ and by $B(G)$ the subgroup of $G$ such that $B(G) / \tau(G)$ is the Hirsch-Plotkin radical of $G / \tau(G)$. It is easy to see that $B(G)$ is a normal subgroup of $G$.

Proposition 1. Let $D$ be a division ring with center $F$. If $G$ is a subnormal subgroup of $D^{*}$, then $B(G)$ is contained in $F$.

Proof. Being a normal subgroup of $G$, the subgroup $\tau(G)$ is a periodic subnormal subgroup of $D^{*}$. With reference to [7, Theorem 8], we conclude that $\tau(G)$ is contained in $F$. 
Our next step is to assert that $B(G)$ is indeed a locally nilpotent group. For this purpose, we take an arbitrary finitely generated subgroup $H$ of $B(G)$, and our aim is to show that this is a nilpotent group. It is a simple matter to see that $H \tau(G) / \tau(G)$ is a finitely generated subgroup of $B(G) / \tau(G)$. Accordingly, the local nilpotence of $B(G) / \tau(G)$ implies that $H \tau(G) / \tau(G)$ is nilpotent. We set

$$
\begin{aligned}
& H_{1}=[H, H], \quad H_{2}=\left[H_{1}, H\right], \\
& H_{3}=\left[H_{2}, H\right], \ldots
\end{aligned}
$$

Now, as $H \tau(G) / \tau(G)$ is nilpotent, we can find an integer $n$ for which $H_{n} \subseteq \tau(G) \subseteq F$. This fact says that any element of $H_{n}$ commutes elementwise with $H$ and, in consequence, we have $H_{n+1}=\left[H_{n}, H\right]=1$, from which it follows that $H$ is nilpotent. In other words, we obtain that $B(G)$ is locally nilpotent, as asserted.

As we have pointed out before, $B(G)$ is a normal subgroup of $G$. This assures us to conclude that $B(G)$ is a locally nilpotent subnormal subgroup of $D^{*}$. By virtue of Huzurbazar's result ([8]), we finally obtain that $B(G) \subseteq F$. Our proof is finished.

The following lemma, which provides the key to later success, gives us a way to calculate the normalizer of a locally solvable subgroup in a division ring.

Lemma 2 ([15, Point 20]). Let $R=F[G]$ be an algebra over the field $F$ that is a domain. If $G$ is a locally solvable, then $R$ is an Ore domain. Moreover, if we assume that $D$ is the skew field of fractions of $R$ and that $B(G)=F^{*} \cap G$, then $N_{D^{*}}(G)=G F^{*}$.

Lemma 3. Let $D$ be a division ring with center $F$. If $G$ is a locally solvable non-central subnormal subgroup of $D^{*}$, then $F(G)=D$ and $N_{D^{*}}(G)$ is locally solvable.

Proof. With reference to the previous lemma, the local solvability of $G$ assures us to conclude that $R=F[G]$ is an Ore domain. Accordingly, its skew field of fractions is exactly $F(G)$, the division subring of $D$ generated by $G$ over $F$. Since $F(G)$ contains $G$ which is assumed to be non-central, in the light of Stuth's Theorem $([13$, Theorem 1]), we obtain that $F(G)=D$.

Next, we argue that $B(G)=F^{*} \cap G$. First, it follows directly from Proposition 1 that $B(G) \subseteq F^{*} \cap G$, which implies that $B(G) / \tau(G) \subseteq\left(F^{*} \cap G\right) / \tau(G)$. In regard to the reverse inclusion, we note that, being the Hirsch-Plotkin radical of $G / \tau(G)$, the factor group $B(G) / \tau(G)$ is the largest locally nilpotent normal subgroup of $G / \tau(G)$. On the other hand, it is clear that $\left(F^{*} \cap G\right) / \tau(G)$ 
is an abelian normal subgroup of $G / \tau(G)$, which yields that $\left(F^{*} \cap G\right) / \tau(G) \subseteq$ $B(G) / \tau(G)$. In other words, we must have $\left(F^{*} \cap G\right) / \tau(G)=B(G) / \tau(G)$, from which it follows that $B(G)=F^{*} \cap G$. Our argument is now finished. Finally, the last assertion follows immediately from the proceeding lemma.

Before presenting the main theorem, we need a result of Zalesskii:

Lemma 4 ([17]). Let $D$ be a division ring with center $F$. If $G$ is locally solvable normal subgroup of $D^{*}$, then $G$ is contained in $F$.

Here now is the main results of this section.

THEOREM 1. Let $D$ be a division ring with center $F$. If $G$ is a locally solvable subnormal subgroup of $D^{*}$, then $G$ is contained in $F$.

Proof. There is nothing to prove if $D$ is commutative. Therefore, we may suppose that $D$ is non-commutative. Assume that $G$ is not contained in $F$. Since $G$ is a subnormal subgroup of $D^{*}$, there exists a finite chain of subgroups

$$
G=G_{r} \leq G_{r-1} \leq \cdots \leq G_{0}=D^{*},
$$

in which $G_{i}$ is normal in $G_{i-1}$ for $0 \leq i \leq r$. By virtue of Lemma 3, we conclude that $N_{D^{*}}(G)$, the normalizer of $G$ in $D^{*}$, is a locally solvable group. The normality of $G_{r}$ in $G_{r-1}$ implies that $G_{r-1}$ is contained in $N_{D^{*}}(G)$ and, in consequence, the subgroup $G_{r-1}$ is locally solvable and non-central.

Repeat this procedure, now starting with $G_{r-1}$, we obtain that $G_{r-2}$ is locally solvable, too. This process must eventually terminate after finite steps, and at the final stage, we have the fact that $D^{*}$ is locally solvable. It follows immediately from Lemma 4 that $D$ is commutative, which is a contradiction. Our proof is finally completed.

\section{Locally solvable quasinormal subgroups}

We prepare the way by first establishing a few results concerning groups which are radical over subgroups.

Lemma 5 ([3, Theorem 2]). Let $G$ be a group and $Q$ a quasinormal subgroup of $G$. If $C$ is an infinite cyclic subgroup of $G$ such that $Q \cap C=1$, then $Q$ is a normal subgroup of $Q^{G}$ and $Q / Q_{G}$ is abelian.

Lemma 6. Let $G$ be a group. If $Q$ is a quasinormal subgroup of $G$, then either $G$ is radical over $Q$ or $Q$ is subnormal in $G$ of defect at most 2 .

Proof. To start, we assume that $G$ is not radical over $Q$. As such, we can find an element $g \in G$ such that $g^{n}$ does not belong to $Q$ for every integer 
number $n$. Let $C$ be the cyclic subgroup of $G$ generated by the element $g$. Then, the fact that $g^{n} \notin Q$ for any choice of $n$ ensures that $Q \cap C=1$. By virtue of the above lemma, we obtain that $Q$ is normal in $Q^{G}$, which is a normal subgroup of $G$. Phrased in another way, $Q$ is a subnormal subgroup of $G$ with the correspondent series $Q \unlhd Q^{G} \unlhd G$. This completes our proof.

The next lemma, which is an interesting result of C. Faith, provides the key to establish the main result of this section.

Lemma 7 ([2, Theorem B]). Every division ring which is radical over a proper subring is a field.

By an analogy with C. Faith's result, a ring which is radical over a subgroup may be characterized in the following manner.

Proposition 2. Let $R$ be a ring and $G$ a subgroup of $R^{*}$. If $R \backslash\{0\}$ is radical over $G$, then $R$ is a division ring.

Proof. To prove that $R$ is a division ring, it suffices to show that each nonzero element of $R$ is right invertible. For this purpose, we take $x$ to be an arbitrary nonzero element of $R$. The radicality over $G$ of $x$ permits us to find an integer $n \geq 1$ for which $x^{n} \in G$. As $G$ is a group, we can find an element $g \in G$ such that $x^{n} g=1$. Or, equivalently, we have $x\left(x^{n-1} g\right)=1$. This relation shows that $x$ is right invertible with the right inverse $x^{-1}=x^{n-1} g$. Therefore, the ring $R$ is indeed a division ring and our proposition is proved.

Lemma 8. Let $D$ be a division ring, and $G$ a non-abelian subgroup of $D^{*}$. Assume that $D^{*}$ is radical over $G$. Then, every subring of $D$ containing $G$ is coincided with $D$.

Proof. For a proof by contradiction, we assume that $E$ is a proper subring of $D$ containing $G$. It is a fairly simple matter to see that $E=E[G]$. The assumption on $D^{*}$ assures us to deduce that $D$ is radical over $E$ and so $D$ is a field by Lemma 7. But this contrasts to the fact that $G$ is assume to be non-abelian.

The following theorem illustrates how the multiplicative group of a division ring is affected by certain subgroups over which it is radical.

THEOREM 2. Let $D$ be a division ring, and $G$ a locally solvable subgroup of $D^{*}$. If $D^{*}$ is radical over $G$, then $D$ is a field.

Proof. Suppose, to the contrary, that $D$ is non-commutative. If $G$ is abelian, then $F(G)$ is a proper subfield over which $D$ is radical. It follows from previous lemma that $D$ is a field, which violates our supposition. We 
may therefore assume that $G$ is non-abelian. In the light of Lemma 8, we obtain that $F[G]=D$ and so $G$ possesses an abelian normal subgroup $A$ for which $G / A$ is locally finite ([14, Point 3$])$. This last fact ensures that $G$ is radical over $A$ and, in consequence, so is $D^{*}$. As a result, the division ring $D$ is radical over the subfield $F(A)$, from which it follows that $D$ is a field. Again, we arrive at a desired contradiction, proving our theorem.

This may be a good place to give the main result of this section.

THEOREM 3. Let $D$ be a division ring with center $F$. If $Q$ is a locally solvable quasinormal subgroup of $D^{*}$, then $Q$ is contained in $F$.

Proof. With reference to Lemma 6, we have either $Q$ is subnormal in $D^{*}$ or $D^{*}$ is radical over $Q$. In the first event, our result follows immediately from Theorem 1. It remains to examine the case where $D^{*}$ is radical over $Q$. In this case, previous theorem says that $D$ is commutative, and our result certainly holds. Our proof is now completed.

\section{Acknowledgement}

The authors are profoundly grateful to the referees for their careful reading and suggestions.

\section{References}

[1] L. Q. Danh, M. H. Bien and B. X. Hai, Permutable subgroups in $\mathrm{GL}_{n}(D)$ and applications to locally finite group algebras, arXiv:1912.09887 [math.RA]; to appear in Vietnam Journal of Mathematics.

[2] C. Faith, Algebraic division ring extensions, Proc. Amer. Math. Soc., 11 (1960), 43-53.

[3] F. Gross, Subnormal, core-free, quasinormal subgroups are solvable, Bull. London Math. Soc., 7 (1975), 93-95.

[4] B. X. Hai and D. V. P. Ha, On locally soluble maximal subgroups of the multiplicative group of a division ring, Vietnam J. Math., 38 (2010), 237-247.

[5] B. X. Hai and N. V. Thin, On locally nilpotent subgroups of $G L_{1}(D)$, Comm. Algebra, 37 (2009), 712-718.

[6] R. Hazrat, M. Mahdavi-Hezavehi and M. Motiee, Multiplicative groups of division rings, Math. Proc. R. Ir. Acad., 114A (2014), 37-114.

[7] I. N. Herstein, Multiplicative commutators in division rings, Israel J. Math., 31 (1978), $180-188$.

[ 8 ] M. S. Huzurbazar, The multiplicative group of a division ring, Soviet Math. Dokl., (1960), 1433-1435.

[9] H. V. Khanh, On locally solvable subgroups in division rings, J. Algebra, 247 (2020), $220-225$.

[10] M. Ramezan-Nassab and D. Kiani, Some skew linear groups with Engel's condition, J. Group Theory, 15 (2012), 529-541. 
[11] J. C. Lennox and S. E. Stonehewer, Subnormal subgroups of groups, Clarendon, Oxford, 1987.

[12] S. E. Stonehewer, Permutable subgroups of infinite groups, Math. Z., 125 (1972), 1-16.

[13] C. J. Stuth, A generalization of the Cartan-Brauer-Hua Theorem, Proc. Amer. Math. Soc., 15 (1964), 211-217.

[14] B. A. F. Wehrfritz, Locally nilpotent skew linear group II, Proc. Edinb. Math. Soc., 30 (1987), 423-426.

[15] B. A. F. Wehrfritz, Soluble and locally soluble skew linear groups, Arch. Math. 49 (1987), 379-388.

[16] B. A. F. Wehrfritz, Goldie subrings of Artinian rings generated by groups, Q. J. Math. Oxford, 40 (1989), 501-512.

[17] A. E. Zalesskii, Solvable groups and crossed products, Mat. Sb. (N.S.), 67 (1965), $154-160$.

$$
\text { Le Qui Danh }
$$

(1) Faculty of Mathematics and Computer Science

University of Science

Ho Chi Minh City, Vietnam

(2) Vietnam National University

Ho Chi Minh City, Vietnam

(3)Department of Mathematics Mechanics, and Informatics

University of Architecture Ho Chi Minh City

196 Pasteur Str., Dist. 3, HCM-City, Vietnam

E-mail: danh.lequi@uah.edu.vn

Huynh Viet Khanh

(1) Faculty of Mathematics and Computer Science

University of Science

Ho Chi Minh City, Vietnam

(2) Vietnam National University

Ho Chi Minh City, Vietnam

E-mail: huynhvietkhanh@gmail.com 